\title{
Teaching research of Computer Organization Principles for Emerging Engineering Education
}

\author{
Zhimeng Liu ${ }^{1,2, *}$, Yanli Zhao ${ }^{1}$ \\ ${ }^{1}$ School of Computer Science and Technology, Shandong Institute of Business and Technology, Yantai, \\ China \\ ${ }^{2}$ Key Laboratory of Intelligent Information Processing in Universities of Shandong, Yantai, China \\ *Zhimeng Liu: 2390804676@qq.com
}

Keywords: New Engineering Education, Guided Teaching, Case Teaching, Combined Teaching With Practising.

\begin{abstract}
In view of the new engineering education movement background, the computer composition principle course of network engineering major put forward the new requirements of less hours and knowledge to be refined, the course research team from the requirements of the professional knowledge system, combed the relevance of the knowledge points of each chapter, and designed a guided case-based and combined teaching with practising methods, and implemented in the computer composition principle course teaching process. This paper shows the concrete implementation of the teaching method in the curriculum by taking the teaching of cache-main memory address mapping as an example.
\end{abstract}

\section{Introduction}

New Engineering Education puts forward the new requirement of changing curriculum teaching concept, breaking through traditional teaching barriers, enriching teaching methods and stimulating inner learning interest, which provides a new way of thinking for curriculum teaching reform [1]. Computer composition principle as the core professional course of network engineering, in the whole professional personnel training system plays an important role. This course explains the theoretical knowledge of the model machine, but does not involve specific models and hardware models, the content of the course is relatively abstract, and the working principles of the components are complex and different. Learning objectives require students to be able to link the theoretical knowledge of each component and establish a systematic concept of the whole machine [2]. There are many knowledge points and relatively independent content between the chapters of the curriculum, resulting in the traditional way of teaching often difficult to teach and difficult to learn the dilemma.

In order to solve this difficult problem, under the background of the new engineering education, the course teaching research team, starting from the practical requirements of the applied undergraduate professional training system, based on the teaching experience accumulated over the years, explores a teaching method based on the guiding case teaching method, teaching and practice combination, and applies it to the practical teaching of the computer composition principle course.

\section{The teaching methods}

In the teaching reform of computer composition principle, the curriculum teaching research group applies the teaching mode combining guided teaching with case-based teaching and practice to teaching practice.

\subsection{Guided teaching}

Guided teaching method is a kind of teaching method that pursues the coordination and resonance of teachers and students' thinking activities in teaching activities, stimulates students' enthusiasm for learning, can fully mobilize students' initiative and enthusiasm for learning, and makes the teaching 
process achieve the perfect combination of teaching and learning in a relaxed and pleasant atmosphere, and finally achieve the teaching goal [3].

In the process of teaching the principle of computer composition, the course team selected "Computer Composition Principle" (2nd edition) written by Professor Tang Shuofei as a teaching material, teaching content from the overall framework of the computer hardware system, from top to bottom, from the table and inside, layer by layer refinement, in-depth analysis of the structure and working principles of the major components of computer hardware, and through the system bus to interconnect the major components into a whole machine. Therefore, although the knowledge points of each chapter are relatively independent in the distribution of the contents that make up the whole machine, some knowledge points in the chapters have the correlation before and after. It is this correlation that provides theoretical support for the feasibility of the curriculum group to implement guided case teaching and teaching combined with teaching. Figure 1 shows the guiding process and related knowledge points in Cache teaching.

In guided teaching, the curriculum teaching research group begins by asking the question of "the reason for the introduction of Cache" and strengthens its grasp of this knowledge point by reviewing and discussing the role of the main memory-cache hierarchy in the hierarchy of memory. Then throw the question "Is it feasible to introduce Cache and why?" "The local principle of program access" was introduced after participating in the discussion, which provided theoretical support for the introduction of Cache. This is followed by the introduction of concepts such as "hit and off" by throwing out the new question "How the CPU accesses the information in the main memory through Cache". Throw the new question "Which address does the CPU take information from Cache" again, introducing address mapping knowledge points between Cache and main memory. Finally, by throwing out the question "How does mapping when Cache is full" introduce "replacement algorithm, write consistency" and other related knowledge points.

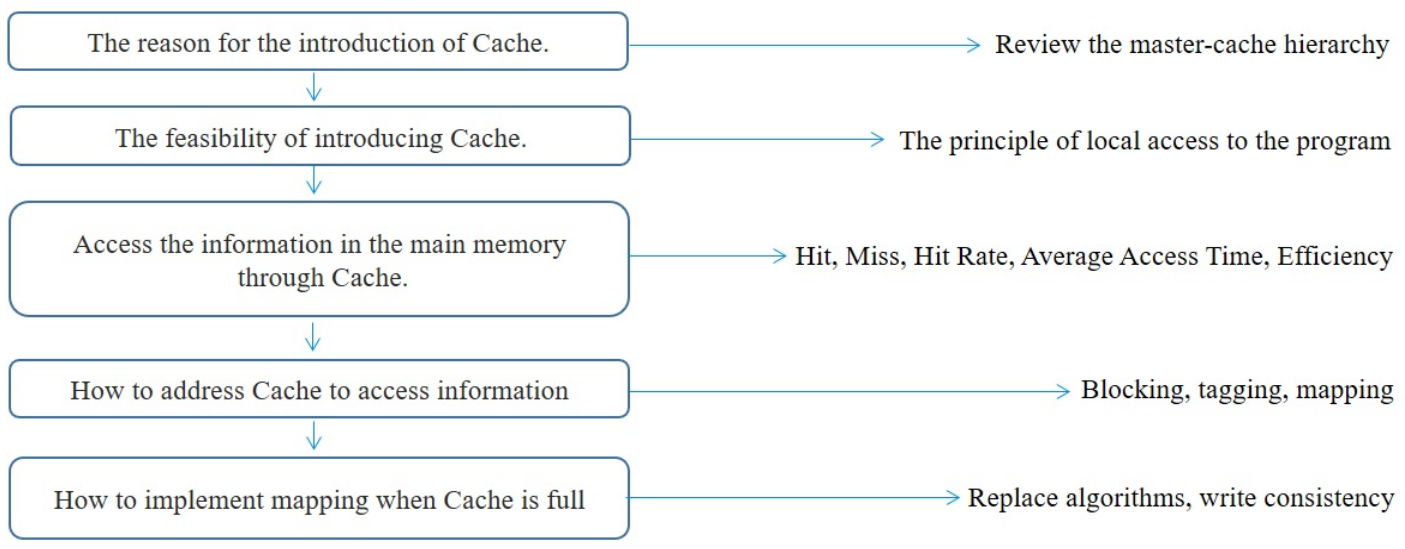

Figure 1. Cache's guided teaching structure diagram

This kind of guided teaching fully mobilizes the enthusiasm of students to ask questions and the initiative to think about them, activates the teaching atmosphere, and enhances students' understanding and mastery of in-depth knowledge points at all levels.

\subsection{Case-based teaching methods combined with practice}

Guided teaching allows students to understand and master the relevant knowledge points and their relevance [4-5]. In order to further strengthen the students' mastery of knowledge points, the curriculum teaching research group has carefully designed teaching cases that include comprehensive knowledge points in the course of guided teaching, which can realize the role of knowledge explanation from small to large, from simple to complicated, from easy to difficult, and through the combination of speaking and practicing, and in the process of learning, students can not only master various knowledge points but also understand the role of knowledge points in the case. This kind of case-by-case, teaching and practice combined teaching method not only shows the application of knowledge points to students, but also understands the degree of knowledge points in time. Figure 2 
below shows designing teaching cases for knowledge points during cache teaching.

Guided teaching Case-by-case of knowledge points

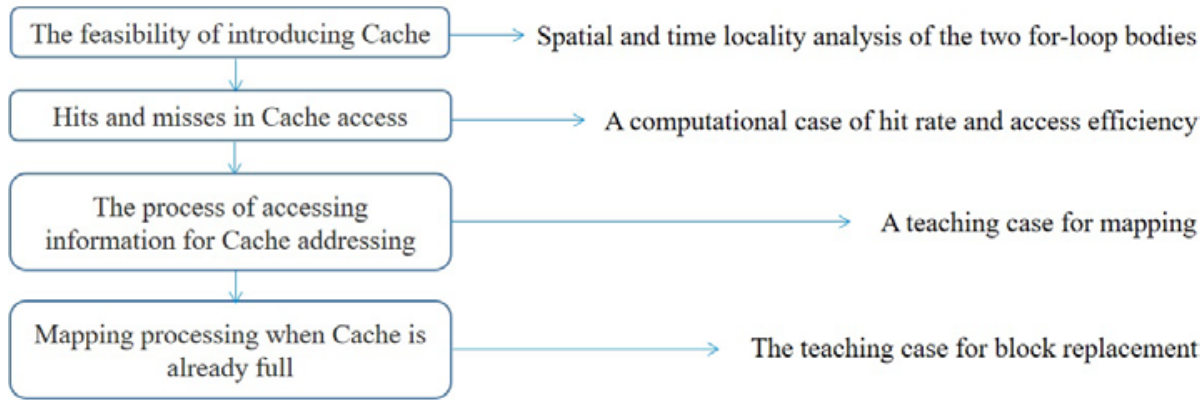

Figure 2. Knowledge point case design diagram in guided teaching

\section{Application of teaching methods in master-Cache address mapping}

Case-based teaching and practice combined teaching mode can integrate the relevant knowledge points of the course, through the layer-by-layer stacking of knowledge points can form a larger knowledge block, the curriculum teaching research group in the chapter of the knowledge block as a unit, carefully designed the knowledge points layer-by-layer contained teaching cases and in the course of teaching to strengthen the understanding of knowledge points. In this section, the Cache-Storage direct mapping method is used as an example to introduce the specific implementation process of this teaching method.

\subsection{Block processing of the main memory and Cache}

In a computer where Cache is configured, a dedicated data path is set between the primary memory and Cache. In order to improve the speed of information transmission between the primary memory and Cache, information is usually transmitted between the main memory and Cache in blocks, so the premise of address mapping between the two is block processing. Relevant knowledge points include how to block, the main memory address format, etc.

To achieve a map with Cache, both the main memory and Cache are divided into blocks of the same size, each containing the same number of words. Then, the main memory address consisting of $2 \mathrm{~m}$ addressable words is divided into a high m-bit primary memory address and a low b-bit in-block address. The same is done for Cache, whose addresses are divided into high c-bit cache block number fields and low b-bits that represent addresses within the block, and ensure that the main memory is the same size as Cache's block size. Block processing allows the primary address to be divided into two segments of the primary block address and the number of address digits within the block.

\subsection{Block case teaching}

Suppose the machine word length is 16 bits, each block contains 4 words, Cache has a total of 8 words, the main memory has a total of 32 words, then calculate the values of b, c, m. Figure 4 below shows.

\subsection{Block marker for the main memory}

Because the primary memory capacity is much larger than Cache's capacity, multiple master blocks are mapped to the same block of Cache. Therefore, a marker is added to represent the primary memory corresponding to the current Cafe, the content of which is equivalent to the high $\mathrm{m}$ bit (full-phase mapping) or part of the main memory (direct mapping and group-linked mapping, bit length equal to m minus the number of digits of the Cache block). Note that a valid bit that should be added here to indicate whether the content contained in the corresponding Cache block makes sense. 


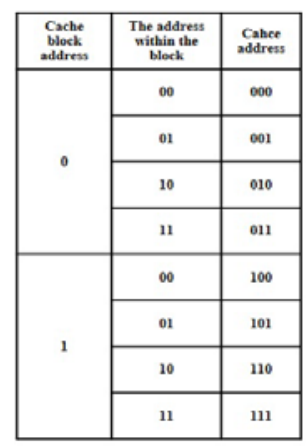

\begin{tabular}{|c|c|c|}
\hline $\begin{array}{l}\text { The primary } \\
\text { block address }\end{array}$ & $\begin{array}{c}\text { The addrens } \\
\text { within the } \\
\text { block }\end{array}$ & $\begin{array}{c}\text { The primary } \\
\text { saddress }\end{array}$ \\
\hline \multirow{4}{*}{000} & 00 & 00000 \\
\hline & 01 & 00001 \\
\hline & 10 & 00010 \\
\hline & 11 & 00011 \\
\hline \multirow{4}{*}{001} & 00 & 00100 \\
\hline & 01 & 00101 \\
\hline & 10 & 00110 \\
\hline & 11 & 00111 \\
\hline \multirow{4}{*}{010} & 00 & 01000 \\
\hline & 01 & 01001 \\
\hline & 10 & 01010 \\
\hline & 11 & 01011 \\
\hline \multirow{4}{*}{011} & 00 & 01100 \\
\hline & 01 & 01101 \\
\hline & 10 & 01110 \\
\hline & 11 & 01111 \\
\hline \multirow{4}{*}{100} & 00 & 10000 \\
\hline & 01 & 10001 \\
\hline & 10 & 10010 \\
\hline & 11 & 10011 \\
\hline \multirow{4}{*}{101} & 00 & 10100 \\
\hline & 01 & 10101 \\
\hline & 10 & 10110 \\
\hline & 11 & 10111 \\
\hline \multirow{4}{*}{110} & 00 & 11000 \\
\hline & 01 & 11001 \\
\hline & 10 & 11010 \\
\hline & 11 & 11011 \\
\hline \multirow{4}{*}{111} & 00 & 11100 \\
\hline & 01 & 11101 \\
\hline & 10 & 11110 \\
\hline & 11 & 1111 \\
\hline
\end{tabular}

Figure 4. Main memory and cache are chunked

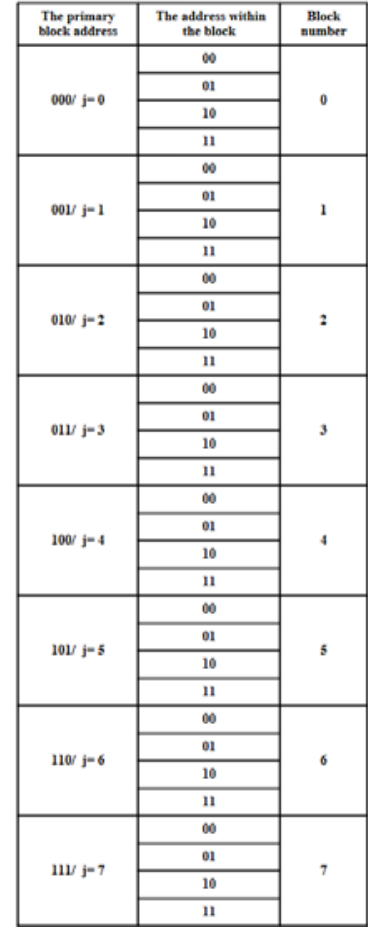

Figure 5. Block tag

In this way, the primary address can be divided into three fields, the primary block marker bit, the primary block address bit and the in-block address bit, as shown in Figure 5.

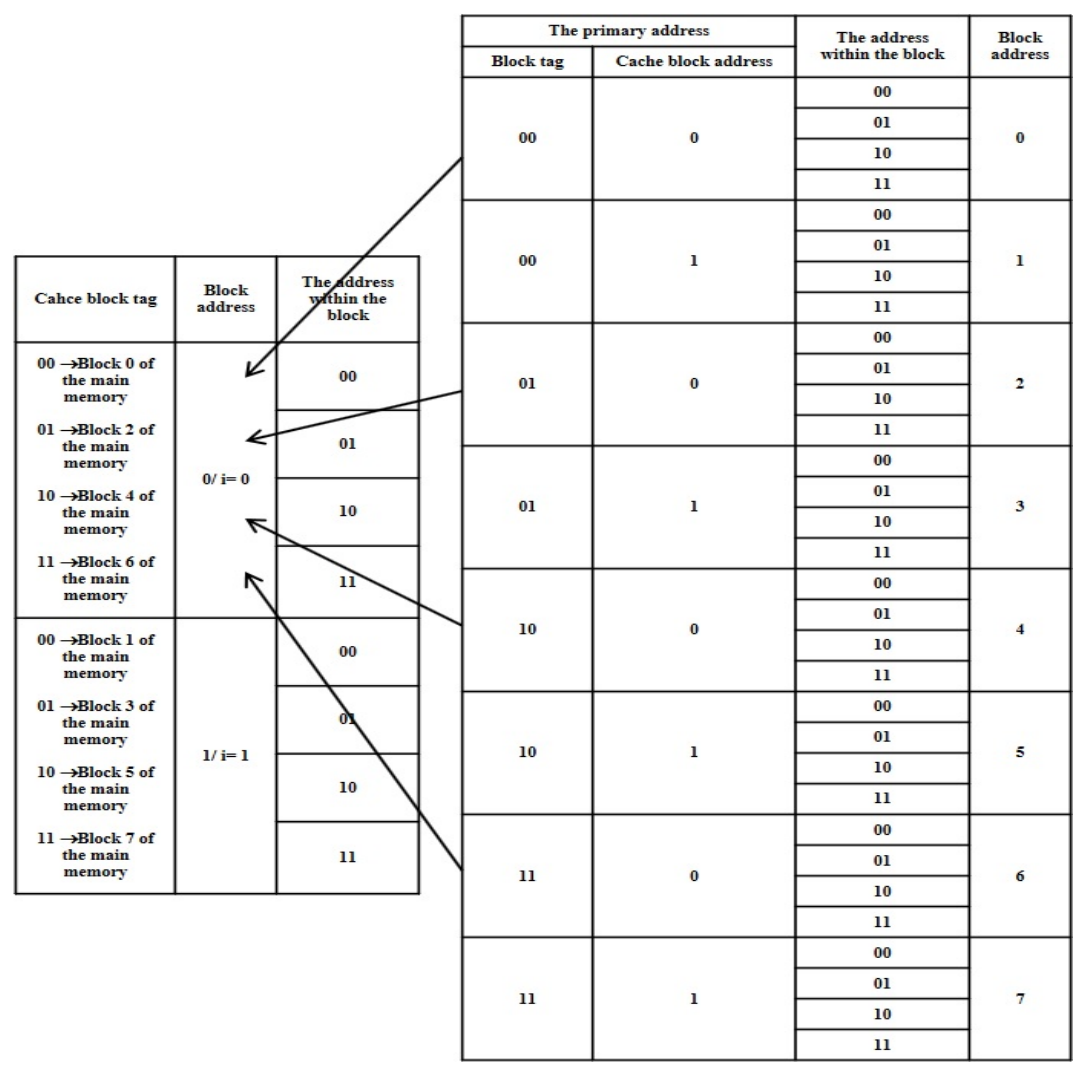

Figure 6. Direct mapping

\subsection{The address mapping method of the primary memory and Cache}

To ensure the correctness of the main memory and cache exchange block data, certain mapping rules must be followed between the mapping blocks, so that when the CPU wants to access a master memory unit word, it can look for the information to be accessed in the block corresponding to cache 
according to the mapping rules instead of the entire cache. Depending on the mapping relationship, cache and master address mapping include full-link mapping, direct mapping, and group-linked mapping. In the teaching of direct mapping, the guided teaching shown in Figure 6, and the case-based teaching method of teaching combined with practice shown in Figure 7 are used to guide students to think and solve problems.

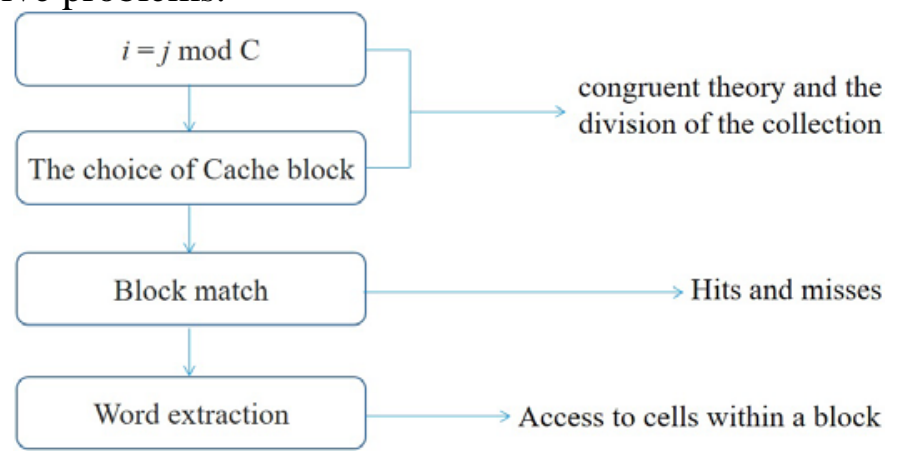

Figure 7. Knowledge-oriented diagrams

Finally, in order to check the students' understanding and mastery of this knowledge point, the course teaching research group tested the students online on the teaching platform "classroom school" in the case of direct mapping cache in operation provided in literature [5], and the test results reflected that most students can better understand and master the working principle of direct mapping.

\subsection{Teaching design of main memory-Cache address mapping}

On the basis of carefully combing the knowledge points of the chapters and their interrelations, the curriculum teaching research group designed the problem guidance map of the knowledge block, the relevant knowledge points and the teaching case combining teaching and practice, and finally implemented the knowledge block as the basic module in the teaching practice. The scenario design process for the knowledge block is shown in Figure 8, and the design process of teaching program combining teaching with practice in direct mapping is shown in Figure 9.

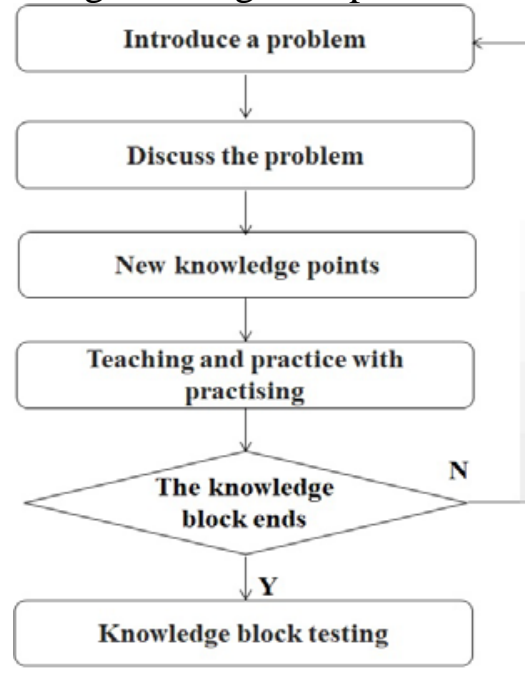

Figure 8. The design of knowledge blocks

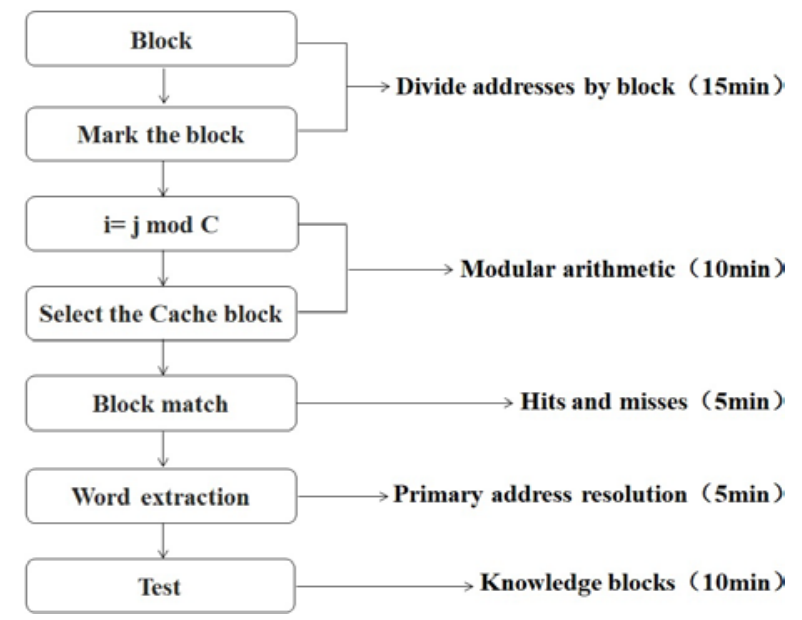

Figure 9. The process of direct mapping

\section{Conclusions}

Under the background of new engineering education, in order to fully mobilize students' enthusiasm, initiative and participation in learning, so that most students can understand and master the key points and basic applications of knowledge in limited class hours, and achieve better teaching results, the curriculum teaching research team studied the combined teaching mode based on guided case-based teaching and practice, and completed the case design of knowledge blocks. 
After several rounds of teaching practice in network engineering, the expected teaching goal of teaching reform has been achieved. The next step for the Curriculum Teaching Research Group will be to begin to design relevant cases and implementation for knowledge surfaces consisting of multiple knowledge blocks.

\section{Acknowledgements}

We would like to thank the financial support from Teaching Reform Research Project of Shandong Technology and Business University (Grant No. 11688G201713).

\section{References}

[1] Cheng, W.Z., Wan, L. A discussion on the reform of guided teaching for new engineering, Journal of Hunan Institute of Science and Technology, vol.39, no.10, pp.21-22, 2018.

[2] T. Shuofei, The principle of computer composition, 2rd ed., Beijing: Higher Education Press, 2015, pp.136-146.

[3] Y. Chun Feng, The foundation of the computer system, Beijing: Machinery Industry Press., 2017, pp. 271-350.

[4] L. Yao, The principle of computer composition, 2rd ed., Beijing: Tsinghua University Press, 2012, pp.231-137.

[5] Bryant R E. O 'Hallaron D R. Computer systems: A programmer's perspective, Upper Saddle River: Prentice Hall, 2003, pp.579-647. 